\title{
Multispectral Plant Health Imaging System for Space Biology and Hypobaric Plant Growth Studies
}

Talal Abboud ${ }^{1,2, *}$, Alain Berinstain ${ }^{1}$, Matthew Bamsey ${ }^{3}$, Robert Ferl ${ }^{3,5}$, Anna-Lisa Paul ${ }^{3}$,
Thomas Graham $^{4}$, Mike Dixon ${ }^{4}$, Demos Leonardos ${ }^{4}$, Michael Stasiak ${ }^{4}$, and Rita Noumeir ${ }^{2}$

1 Space Science and Technology, Canadian Space Agency, 6767 route de l'aéroport, Longueuil, QC J3Y8Y9, Canada;

2 École de technologie supérieure, 1100 rue Notre-Dame O, Montréal, QC H3C1K3, Canada;

3 Horticultural Sciences, University of Florida, Gainesville, FL 32601, USA;

4 Controlled Environment Systems Research Facility, School of Environmental Sciences, University of Guelph, 50 Stone Road East, Guelph, ON N1G2W1, Canada;

5 Interdisciplinary Center for Biotechnology Research, University of Florida, Gainesville, FL 32610, USA

* Author to whom correspondence should be addressed; E-Mail: talal.abboud.1@ens.etsmtl.ca

Received: 11 April 2013 / Accepted: 16 May 2013 / Published: 03 June 2013

\begin{abstract}
Ensuring high plant yields is critical for the application of higher plants to spacebased biological life support systems. An imager capable of monitoring several fluorescent biological markers in real-time would provide a robust health monitoring system and allow growers to minimize environmental stressors. This article reports the results of the design and development of a multispectral plant health imager prototype, employed in a lowpressure plant growth chamber as a test of its functionality in spaceflight systems. Images were collected every four hours with a monochromatic camera and a commercial liquid crystal tunable filter. This arrangement permitted the monitoring of emission from introduced green fluorescent proteins as well as chlorophyll fluorescence. Data were saved locally and accessed in real-time from the exterior of the chamber.
\end{abstract}

Keywords: plant health imaging; multispectral imaging; remote sensor; biological life support; space analog. 


\section{Introduction}

Humans face a variety of challenges as they continue to explore beyond the frontiers of planet Earth. Exploration missions are often severely constrained by launch mass and resupply considerations. The use of plants as part of life support systems continues to be explored as an approach for more sustained human presence in space. In particular, bioregenerative life support systems have been considered since the early $20^{\text {th }}$ century [1]. The Canadian Space Agency, University of Florida and University of Guelph have been involved in assessing the possibility of supporting human presence on the Moon and Mars by deploying greenhouses as plant production system testbeds $[2,3]$. The main concept is the use of plants to regenerate the three cornerstones of human consumable requirements; air, water and food [4]. Spaceflight and other extraterrestrial environments provide unique challenges for plant life. There originates the importance of understanding the metabolic issues that can influence plant growth and development in space. Plant monitoring systems with the capacity to observe the condition of the crop in real-time within these systems would permit operators to take immediate action to ensure optimum system yield and reliability. In addition to the utilization of chlorophyll fluorescence, specific stress response genes can be tagged with reporter genes encoding a variety of fluorescent proteins, allowing gene activities, and by extension plant health, to be monitored through the fluorescence of these gene products [5]. The Transgenic Arabidopsis Gene Expression System (TAGES) is a biosensor that uses Arabidopsis thaliana fluorescence information from both naturally occurring chlorophyll red/near infrared fluorescence, as well as green fluorescence originating from the gene products of green fluorescent protein (GFP) reporter genes [6,7]. Several commercial systems are available for imaging and capturing plant fluorescence, but most analytical procedures involve laboratory examination and human input. However, advanced biological experiments on orbit, the Moon, and Mars are likely to be autonomous, precluding any direct human control over the monitoring/imaging systems. Furthermore, if a mission does include a physical human presence, there are still system trade-off considerations between internal greenhouse/growth chamber operating pressure, up-mass and crew time requirements that may still dictate completely robotic and/or autonomous bioregenerative life support systems [8].

A Multispectral Plant Health Imaging System (M-PHIS) would provide a considerable step forward in our capacity to monitor advanced life support crops in an autonomous manner $[6,9,10,11,12]$. This article describes the design and development of a prototype multispectral fluorescent imaging system deployed in a hypobaric plant growth chamber at University of Guelph. The imager was designed primarily for multiband imaging of chlorophyll and protein fluorescence with the design being driven by portability and autonomous functionality considerations. The design was also novel in that it employed a commercially available liquid crystal tunable filter (LCTF) and a custom developed LED board with an independently variable grow light LED array. This prototype imager provided realtime data while it was operated within a low pressure chamber through the use of a controller, a smart camera, and a custom designed and variable outputs grow and excitation light emitting diode (LED) light array. The deployment in a low-pressure chamber represents one of a number of possible space analogue and on orbit deployment scenarios. The results of this work will direct future efforts in this area of research and drive further design improvements. 


\section{Materials and Methods}

The primary improvement of the M-PHIS over previously developed space analogue plant health imagers employed by this research team is its ability to selectively detect fluorescence at user selectable center wavelengths and bandwidths. In particular, the LCTF permits the ability to monitor plant-emitted light at any wavelength between 420 and $730 \mathrm{~nm}$ and at varied bandwidths. A custom developed LED board furthers the imager selectivity through highly controllable grow light output and selection of varied excitation energy and center wavelengths and thus better permits the targeting of individual fluorophores [compounds that can re-emit light upon excitation] of interest.

\subsection{Excitation, Emission and Imaging Capture Requirements}

Green fluorescent protein fluoresces in the green waveband when exposed to light in the blue through ultraviolet wavebands. Many GFP variants exist; however, the S65T variant is utilized in this hardware demonstration and characterization study (the S65T variant will be referred to simply as GFP for the remainder of this paper). GFP emission arises within the green portion of the visible spectrum with a peak at approximately $512 \mathrm{~nm}[6,13]$. However, fluorescence emitted by GFP covers a considerable bandwidth, meaning that useful data is also available on either side of this $\lambda=512 \mathrm{~nm}$ emission peak. It is important to note that GFP's excitation peak is at approximately $\lambda=488 \mathrm{~nm}$ and that this wavelength is very close to the short end of the GFP emission spectrum $[6,14]$. This small separation implies that care must be taken to ensure that the excitation light is sufficiently filtered to guarantee no overlap with the emitted light from the sample. This can be achieved by selecting excitation lights that do not radiate above some specified wavelength, or more easily, by filtering all wavelengths above the selected wavelength; in this case, approximately $493 \mathrm{~nm}$. For GFP fluorescence the image sensor must then be capable of monitoring wavelengths between 502 and $550 \mathrm{~nm}$. Chlorophyll autofluorescence e.g. red $(630-680 \mathrm{~nm})$ and NIR $(>680 \mathrm{~nm})$ can be excited across a broad wavelength region spanning 250 to $675 \mathrm{~nm}$ with excitation peaks at $430 \mathrm{~nm}, 470 \mathrm{~nm}, 600 \mathrm{~nm}$ and $660 \mathrm{~nm}$ [15].

\subsection{Hypobaric Plant Growth Chambers}

In order to simulate spaceflight deployment conditions that the imager may be expected to perform under, a hypobaric chamber was used to isolate the imager and to serve as a platform for long duration operational tests. A canopy scale hypobaric plant growth chamber (Figure 1) at the University of Guelph's (Ontario, Canada) Controlled Environment Systems Research Facility (CESRF) was utilized for the test. The CESRF maintains and operates 20 sealed environment chambers including 14 variable pressure chambers capable of sustaining a near vacuum $(<1 \mathrm{kPa})$ [2]. Of these 20 chambers, five are fully automated canopy-scale hypobaric chambers and measure $1.0 \times 1.8 \times 2.5 \mathrm{~m}$ with a total volume of approximately 4500 liters and providing a plant growth area of $1.5 \mathrm{~m}^{2}$ [16]. The light canopy, irrigation and nutrient control system is outlined in Figure 1A, while the M-PHIS is shown deployed inside the chamber in Figure 1B. 
Figure 1. Canopy-scale hypobaric chambers at the Controlled Environment Systems Research Facility (CESRF), University of Guelph: A) Exterior showing lighting canopy, irrigation and nutrient control system, B) M-PHIS deployed in a CESRF hypobaric plant growth chamber.
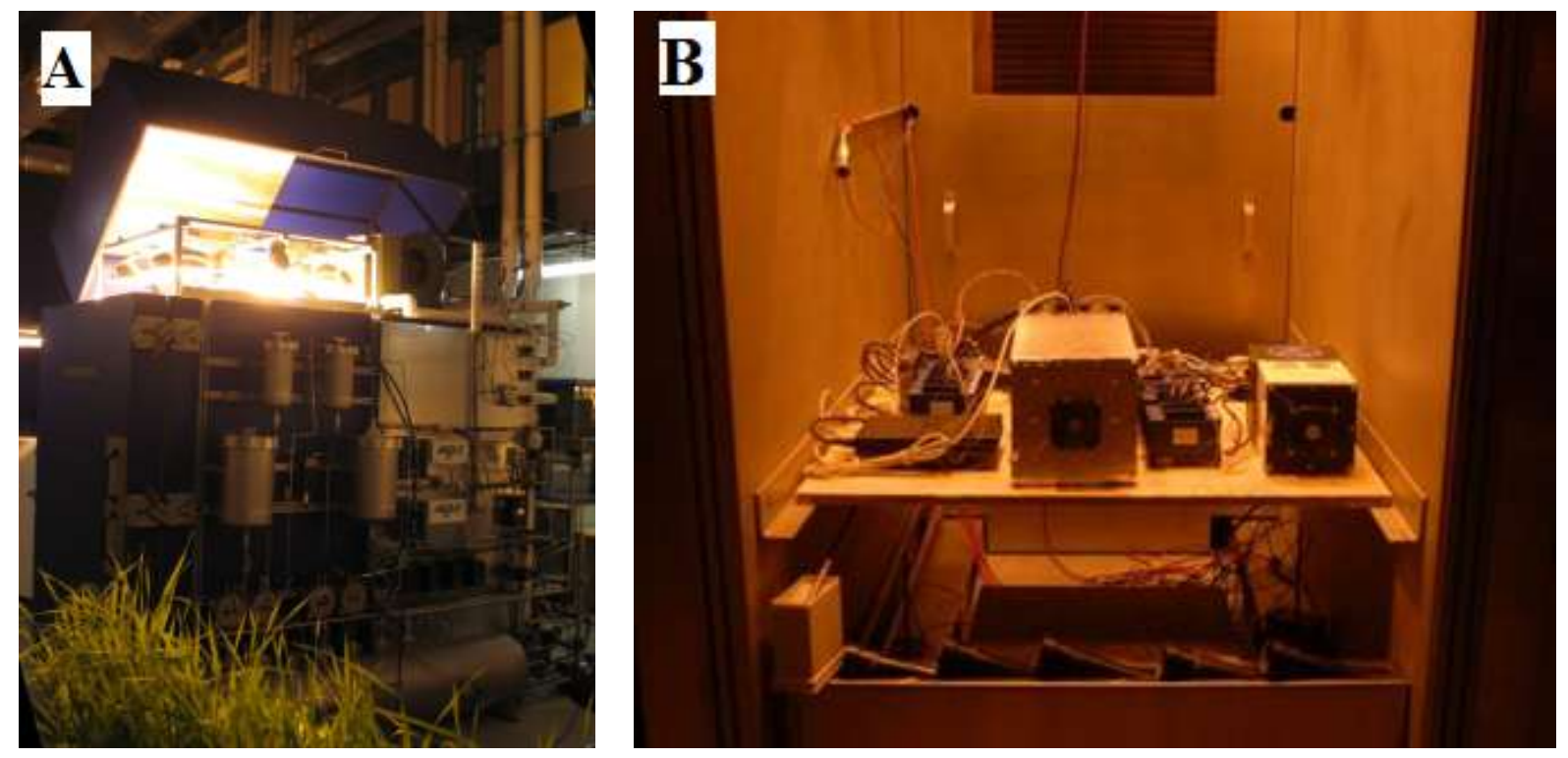

\section{Multispectral Plant Health Imaging System}

The M-PHIS (Figure 2) is a modified version of the TAGES Imaging System-III (TIS-III), which was deployed in the Arthur Clarke Mars Greenhouse in the High Arctic on Devon Island [17]. TIS-III was a modified version of TIS-II, a second generation imaging payload that was designed to collect GFP data in real-time during a spaceflight experiment [7, 18]. The M-PHIS boasts several new features including the capacity to capture images a variety of wavelengths. In addition to capturing GFP expression, M-PHIS could be employed to capture yellow and red fluorescent proteins as well as chlorophyll's red/near infrared florescence and more. In addition, the filter bandwidth could be set by adjusting the LTCF's full width at half max (FWHM). The FWHM is the range of wavelengths (bandwidth) where a filter passes at least $50 \%$ of the light intensity that it passes at the central wavelength. For example, a $510 \mathrm{~nm}$ band-pass filter with a FWHM of $20 \mathrm{~nm}$ will transmit at least $50 \%$ of the light intensity it transmits at this central wavelength between the range 500 to $520 \mathrm{~nm}$. The second most important feature is the independently controllable plant grow light wavelengths. The user has the capacity to set the grow light intensity and the ratio between red and blue wavelengths to match the requirements of the test plants in the system. Given the control over grow light intensity, M-PHIS can also be used to study the influence of different light intensities and red/blue ratio on plant growth. 


\subsection{Hardware}

The multispectral imaging system uses a National Instruments (NI) NI-1744 smart camera as an image capture device (Figure 3). The camera includes a 1280 x 1024 monochrome 8-bit pixel depth CCD, built-in lighting controller, Ethernet ports, and is programmable with NI LabVIEW Real-Time Module or with Vision Builder for Automated Inspection (VBAI). Excitation light is generated by a set of blue LEDs and subsequently filtered by custom sized (65 x $65 \mathrm{~mm}$ with $30 \mathrm{~mm}$ diameter central hole) a $490 \mathrm{~nm}$ short pass filter (Omega Optical) before being incident on the biological sample as shown in Figure 5. Emission filtering is conducted through the application of a Meadowlark TOF-SBVIS LCTF. By utilizing multiple liquid crystal variable retarders and polarizers, the tunable filter is able to select any central wavelength for the passband from 420 to $730 \mathrm{~nm}$. In addition, it permits three different bandwidth sizes to be chosen: narrow, medium and wide. To avoid Kalnienk vision (circular tunnel-like field of vision), due to the physical dimensions of the LCTF (nine centimeter length), a Computar M2514-MP lens with a long focal length of $\mathrm{f}=25 \mathrm{~mm}$ was needed and attached to the camera. This explains the requirement for the long $30 \mathrm{~cm}$ distance between the end of the LCTF and the sample holder, since a closer target resulted in an out-of-focus image. A custom designed bracket attachment held the three elements (camera, lens and LCTF) in line as shown in Figure 3. A local network links different components through an Ethernet hub. These components, namely the imaging system, tunable filter controller, grow lights, white lights, cooling fans, power systems and the data acquisition system, are controlled through NI Compact FieldPoint modules (cFP-2120). NI pulse width modulation (PWM) modules (cFP-PWM-520) controlled the different grow light wavelength intensities, a relay module served as a switch for the camera, cooling fans, white lights and tunable filter controller, and a thermocouple input module acquired temperatures in front and behind the biological sample holder. To simplify the design, 24 VDC components were selected for the build since the NI controller and camera utilized a 24 VDC power supply. The LCTF (Figure 3) is the only component requiring a different voltage. LabVIEW code provided by Meadowlark was integrated into the main imager control software program to permit control of the LCTF though a serial connection to the Compact FieldPoint controller. In addition, a hardware connection was made between the NI-relay and the LCTF controller to control the tunable filter power feed. A small printed circuit board (PCB) containing only white LEDs was positioned directly in front of the biological sample tray, was utilized to illuminate the samples when capturing normal (non-fluorescent) images (Figure 4). The other optical elements of the imager and their respective positions are provided in Figure 5.

The power budget for each component of M-PHIS is displayed in Table 1. As not all components are active at the same time, actual instantaneous power consumption depends on the current state or operational mode of the imager. When the full grow light board is set at $100 \%$ intensity, the total power consumption is approximately $68.12 \mathrm{~W}(54.7 \mathrm{~W}+6.1 \mathrm{~W}+3 \mathrm{~W}(\max )+4.32 \mathrm{~W})$. During a typical capture sequence, which in nominal operations mode occurs once every four hours, the camera and LCTF are powered on for approximately twenty minutes, the excitation LEDs for three minutes and the white light board for one minute. 
Table 1. Power consumption of Multispectral Plant Health Imaging System components.

\begin{tabular}{|l|l|l|l|}
\hline \multicolumn{1}{|c|}{ Component } & \multicolumn{1}{c|}{ Power } & \multicolumn{1}{c|}{ Component } & \multicolumn{1}{c|}{ Power } \\
\hline NI-1744 Camera & $6.48 \mathrm{~W}$ & Ethernet Switch & $\sim 3 \mathrm{~W}$ (Max) \\
\hline cFP-2120 Controller & $6.1 \mathrm{~W}$ & Grow Lights & See Table 3 \\
\hline cFP-PWM-520 Modules & $0-1 \mathrm{~W}(\mathrm{Max})$ & Excitation Lights & $9.64 \mathrm{~W}$ \\
\hline cFP-RLY-421 Module & $0-2.5 \mathrm{~W}(\mathrm{Max})$ & White Light & $\sim 3 \mathrm{~W}$ \\
\hline cFP-TC-120 Module & $0.35 \mathrm{~W}$ & Cooling Fans & $4.32 \mathrm{~W}$ \\
\hline LCTF & $\sim 6 \mathrm{~W}$ & & \\
\hline
\end{tabular}

Figure 2. Multispectral Plant Health Imaging System (M-PHIS) Front (A), Back (B).

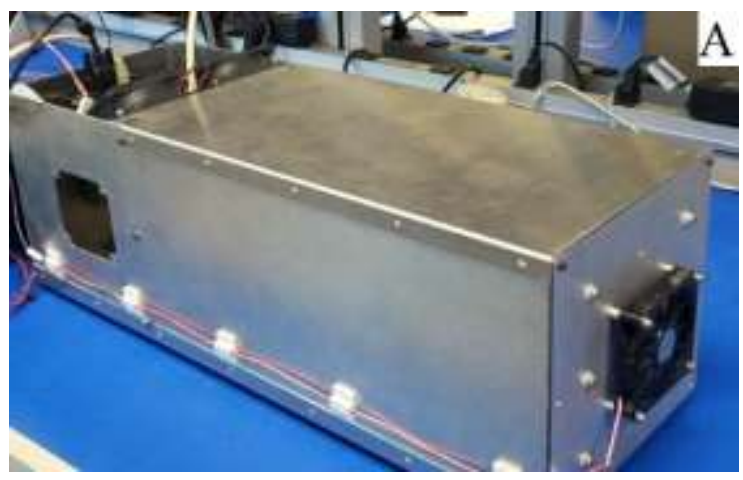

Figure 3. NI-1744 Camera (left), bracket attachment and LCTF (right).

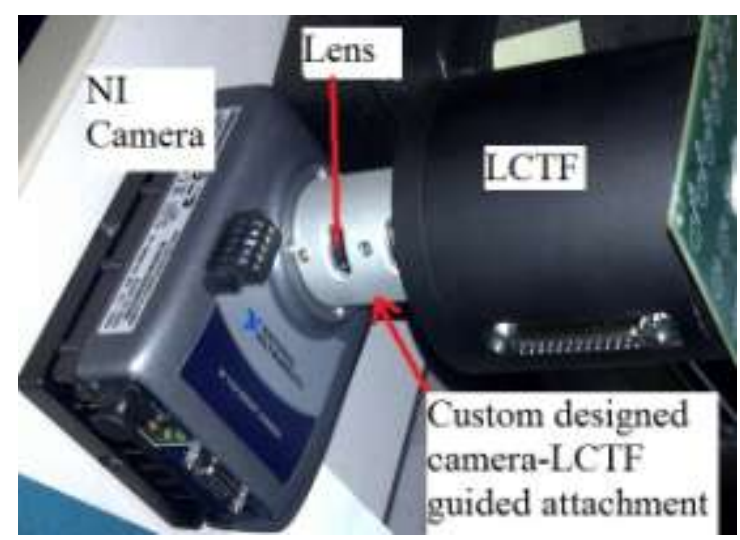

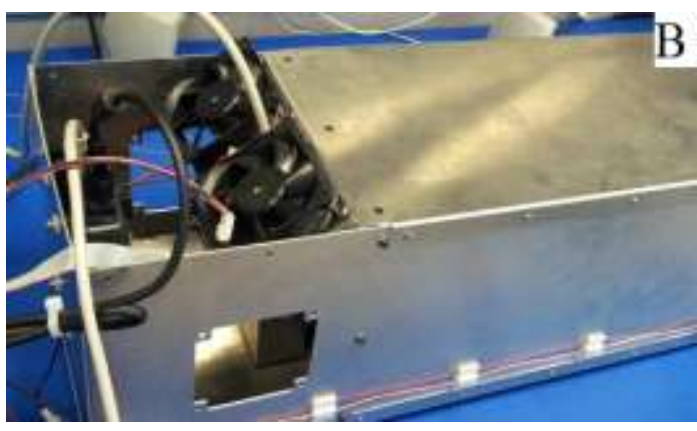

Figure 4. White LEDs and biological sample holder showing installed Petri dish with calibration targets.

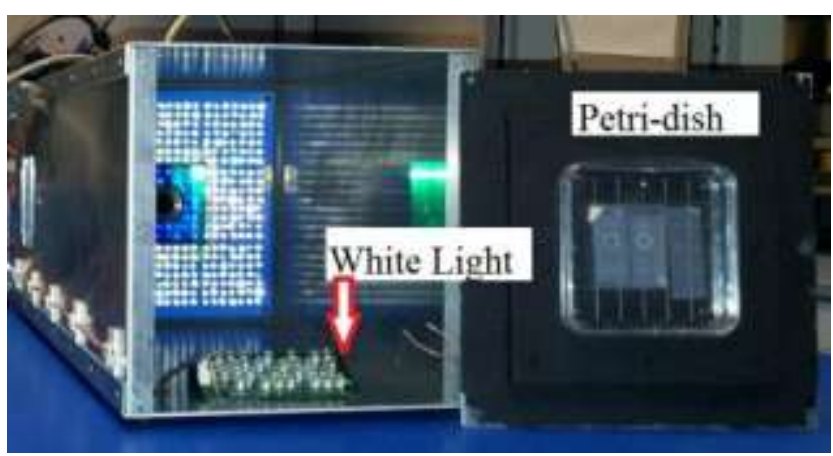


Figure 5. Multispectral Plant Health Imaging System (M-PHIS) with top cover removed and internal items shown.

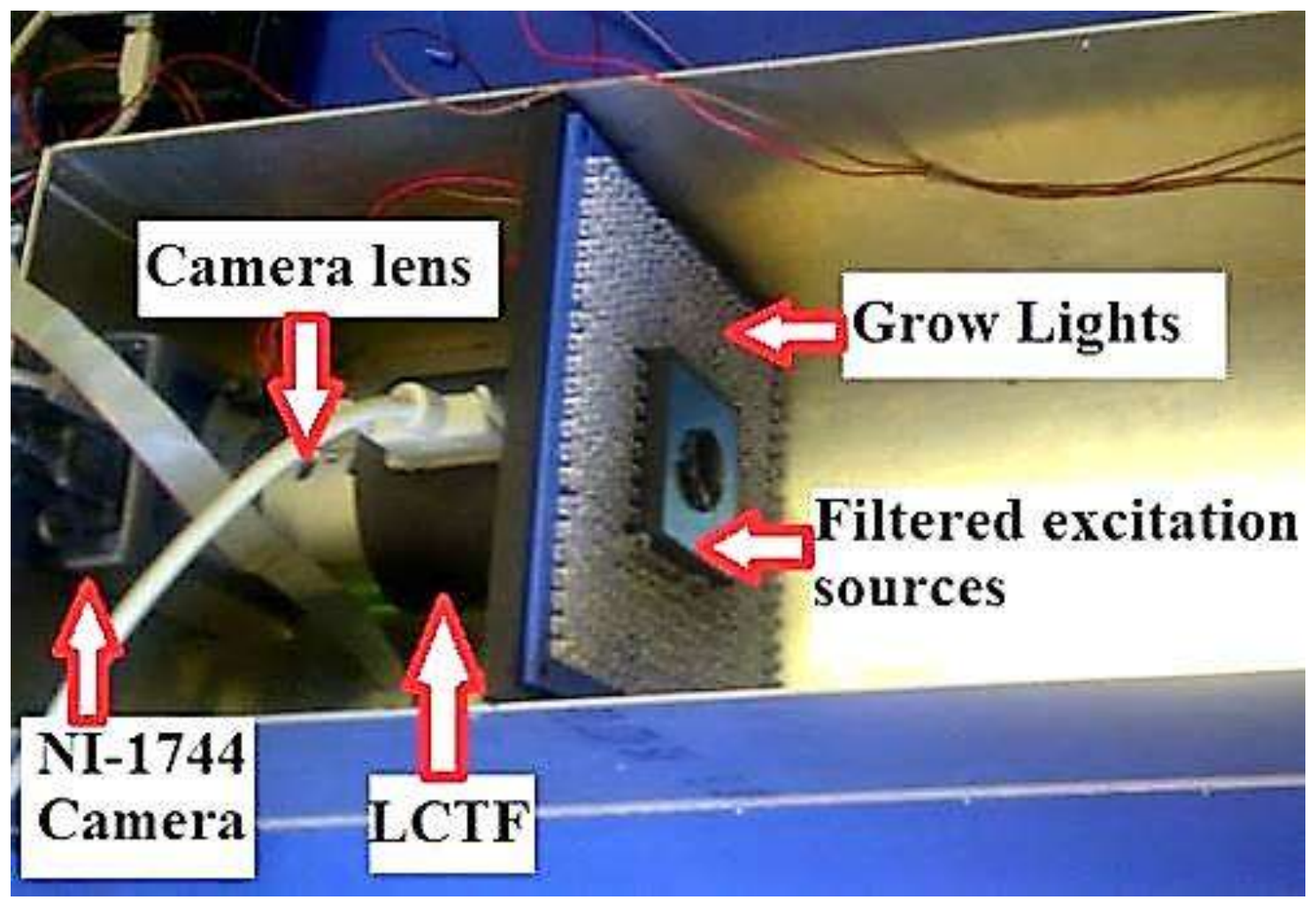

\subsection{Grow Lights}

The design of an LED grow light / excitation light board built from the ground up was novel in its ability to have independent control over the intensities of each wavelength. LEDs have long lifetimes, require little maintenance, are relatively energy efficient and are a rapidly advancing technology area. These features make LEDs an increasingly advantageous technology for artificial lighting in plant growth systems [19] and the preferred lighting technology for M-PHIS. Different red and blue LEDs were selected based upon previous studies of their application to plant growth [20, 21, 22, 23]. Three types of red and blue LEDs were chosen based on the peak absorption wavelengths of chlorophyll $a$ and $b$; 430 to $470 \mathrm{~nm}$ and from 630 to $680 \mathrm{~nm}$ respectively. Since green light is primarily reflected by plants, only a small number of green LEDs were incorporated into the grow light board [19]. Laboratory light distribution tests were conducted to find an illumination pattern that was considered the best at illuminating the entire plant growth area when utilizing plants grown in a standard $10 \mathrm{~cm} \mathrm{x}$ $10 \mathrm{~cm}$ rectangular Petri dish as shown in Figure 6 (note: the imager can be modified to incorporate plant samples of other sizes and orientations). Figure 7 and Table 2 present the distribution of MPHIS's primary LED board incorporating both the growth and excitation LED sets. The measured relative irradiance spectra of M-PHIS's LED grow lights with all grow LEDs activated is shown in Figure 8 . 
Figure 6. Arabidopsis thaliana seeds containing reporter genes growing vertically in a $10 \mathrm{~cm}^{2}$ plates.

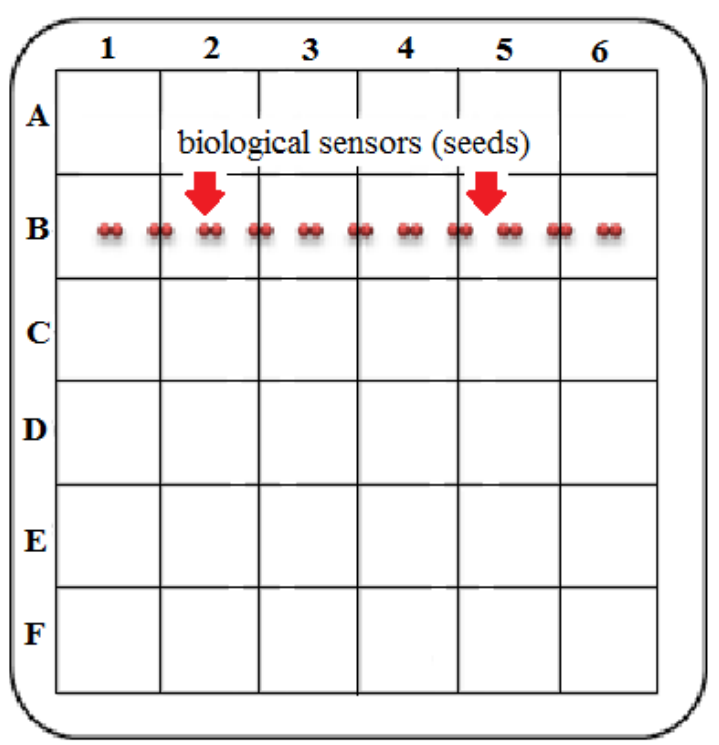

Figure 7. Distribution of grow lights (exterior rectangular region) and excitation lights (interior rectangular region). The centre hole is aligned in front of the LCTF and camera to permit imaging through the LED board.

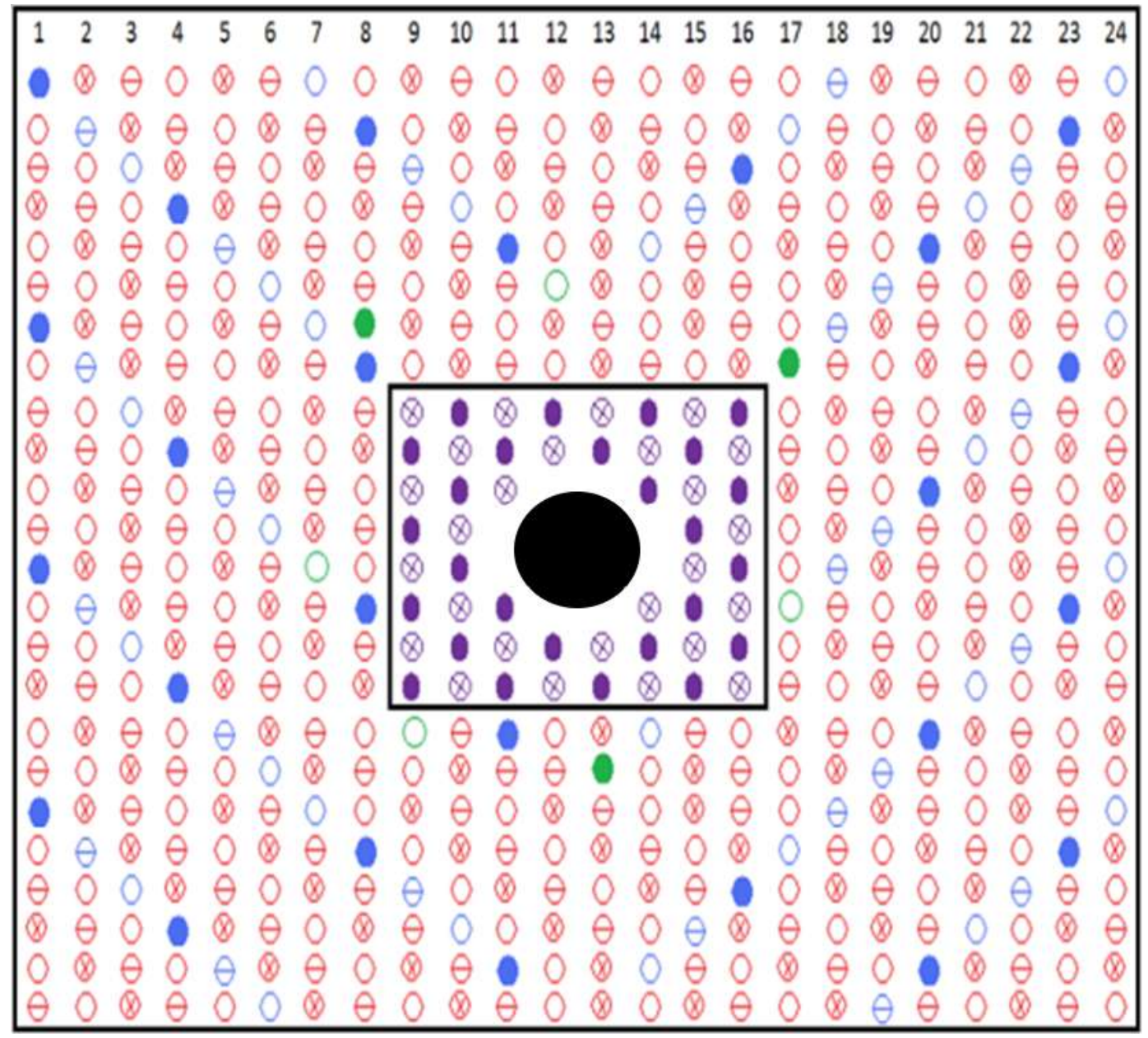


Table 2. Selected Roithner Lasertechnik grow and excitation LEDs, top-level specifications and their quantity within the developed Multispectral Plant Health Imaging System (M-PHIS) LED board.

\begin{tabular}{|c|c|c|c|c|c|}
\hline & LED & $\begin{array}{c}\text { Peak } \\
\text { Wavelength } \\
(\mathbf{n m})\end{array}$ & $\begin{array}{c}\text { Max Voltage } \\
\text { (V) }\end{array}$ & $\begin{array}{c}\text { Max Current } \\
(\mathbf{m A})\end{array}$ & Quantity \\
\hline$\ominus$ & B5b-436-30 & 660 & 2.4 & 30 & 117 \\
\hline$\otimes$ & B5b-435-TL & 630 & 2.5 & 50 & 117 \\
\hline 0 & ELD-670-524 & 680 & 2.2 & 50 & 117 \\
\hline$\ominus$ & LED435-12-30 & 435 & 3.5 & 25 & 21 \\
\hline 0 & B56L5111P & 470 & 3.4 & 50 & 21 \\
\hline 0 & LED450-01 & 450 & 3.4 & 50 & 21 \\
\hline 0 & B5-433-B505 & 500 & 4.0 & 30 & 3 \\
\hline $\mathrm{O}$ & 5G4HCA-H & 520 & 3.8 & 30 & 3 \\
\hline$\otimes$ & LED450-01 & 450 & 4.0 & 50 & 24 \\
\hline 0 & B56L5111P & 470 & 3.8 & 50 & 24 \\
\hline
\end{tabular}

Figure 8. Relative irradiance spectra of the Multispectral Plant Health Imaging System (M-PHIS) primary LED board.

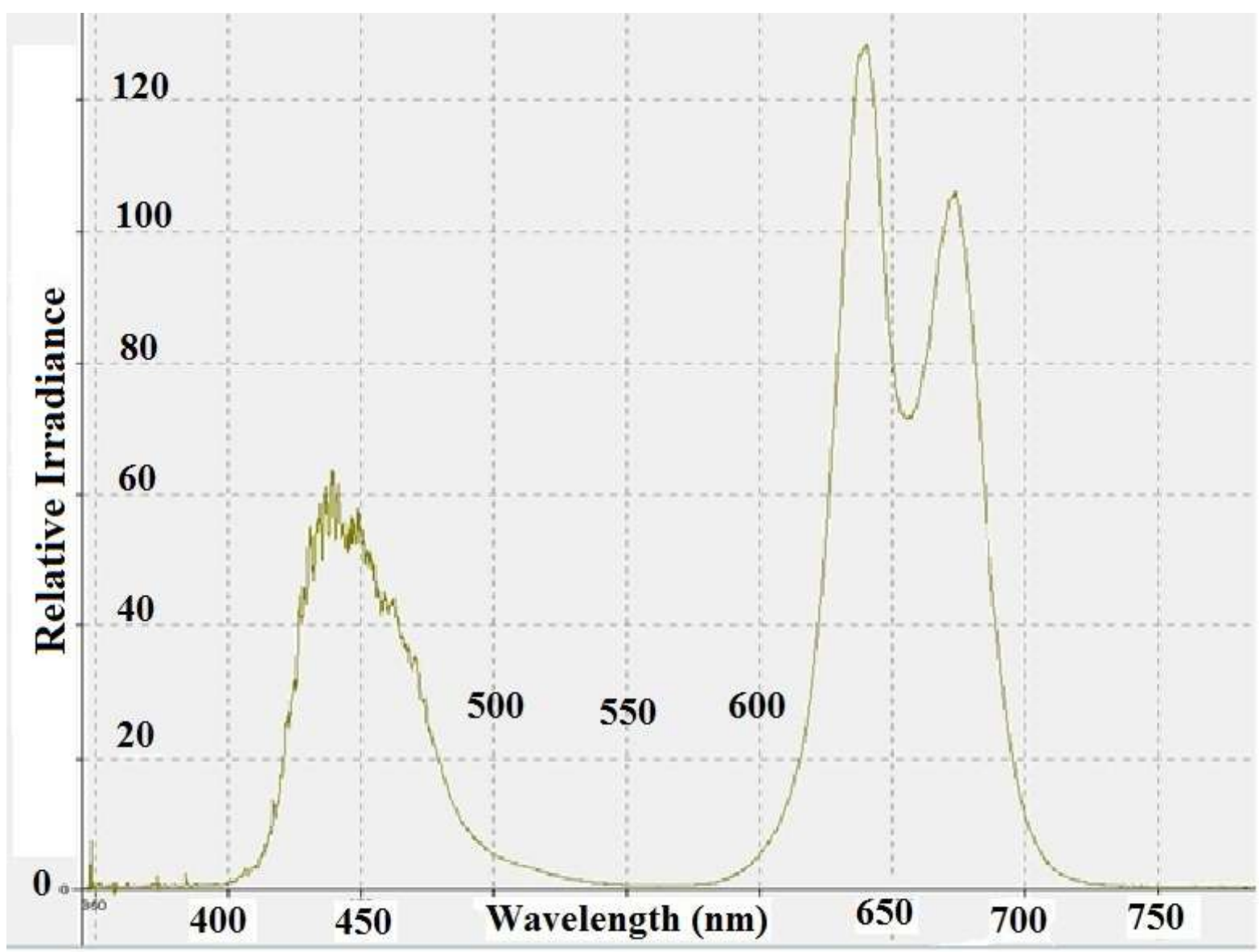


In addition to the various light combinations and ability to tune the output wavelengths, the upper and lower half of the M-PHIS LED board can be independently controlled. This separate control is advantageous because it allows the operator to more easily illuminate the area where the plant's leaves are located while applying less light to the roots where it is typically not required. This can result in energy savings, which is of paramount importance when designing for spaceflight experiments where power is often a limitation. The utilization of fewer LEDs also resulted in a reduction in heat generation and heat management requirements. Figure 9 exhibits several examples of the various possible light combinations and intensity control provided by the LED board.

To better focus the energy emitted by the grow lights on the biological target, a parabolic angle was built into the LED board by employing a custom LED guide produced on a 3D printer (Figure 10). The guide was installed on the circuit board before populating it with LEDs. Grow light LED circuits where designed using series/parallel combination of LEDs with each chain incorporating a ballast resistor in series with the LEDs. Figure 11 provides an example of the described series/parallel arrangement of LEDs for the B5b-435-TL grow LEDs and serves as a useful reference for the power analysis described in the following sections. Resistors were installed on the back of the PCB, outside and away from the biological sample chamber. Cooling fans were located near this back face to aid in dissipating the heat generated by the LEDs.

Figure 9. Grow / excitation light board in operation. A) All grow lights activated at 5\% intensity. B) Upper section, all grow light enabled at 10\%. C) Excitation lights activated at 25\% D) $470 \mathrm{~nm}$ LEDs activated at $5 \%$.

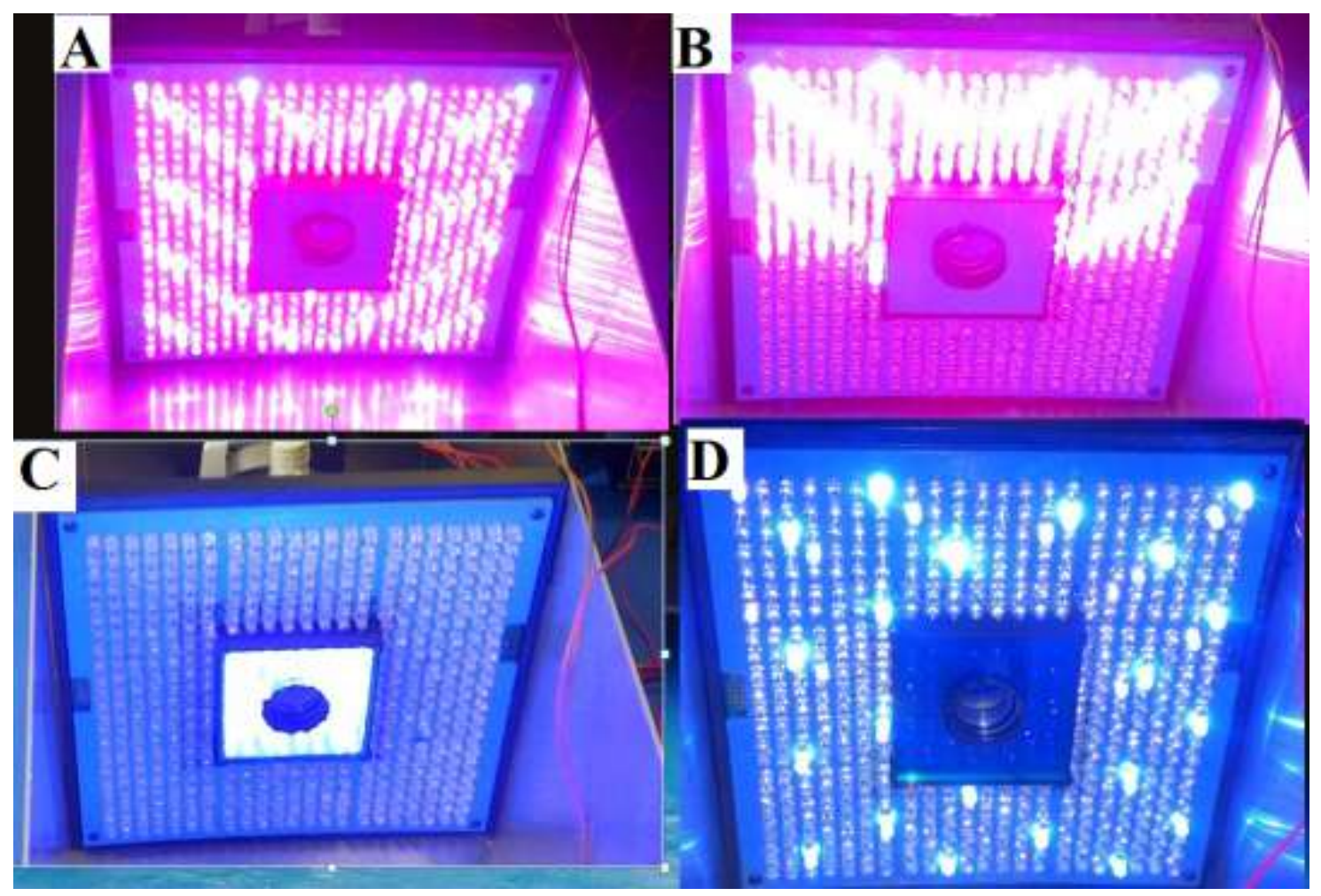


Figure 10. Demonstrating Multispectral Plant Health Imaging System (M-PHIS) grow light board parabolic angle that aids in focusing the energy emitted on the desired target.

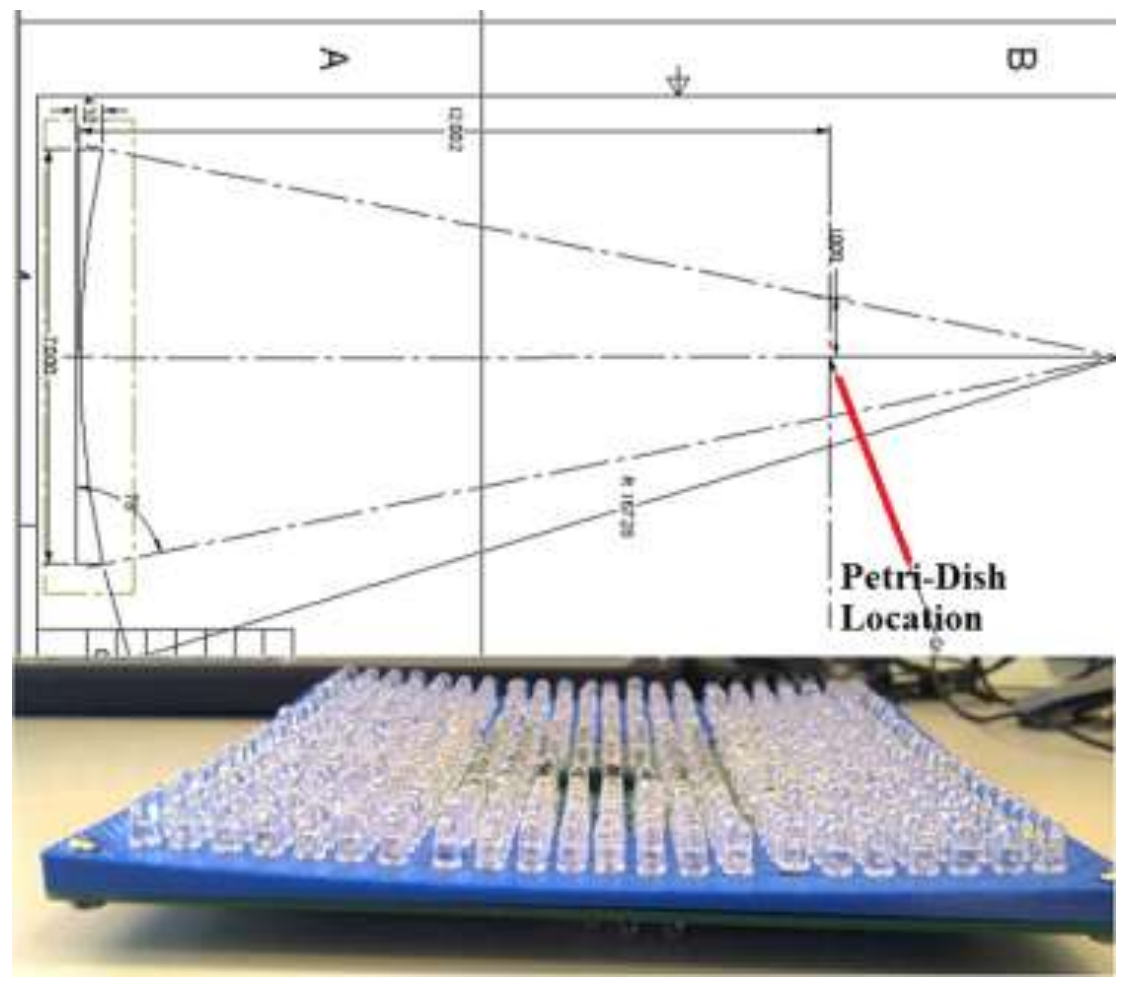

Table 3. Grow light power consumption if the PWM is set to $100 \%$.

\begin{tabular}{|c|c|c|c|c|c|c|c|c|c|c|c|c|c|}
\hline & $\ominus \mathbf{U P}$ & DW & $\otimes U P$ & DW & OUP & DW & ӨUP & DW & OUP & DW & OUP & DW & 00 \\
\hline $\begin{array}{l}\text { Resistors } \\
\text { power (mW) }\end{array}$ & 516.6 & 442.8 & 577.5 & 495 & 1750 & 1500 & 243.75 & 337.5 & 317.5 & 500 & 207.5 & 410 & 773 \\
\hline $\begin{array}{l}\text { Total Pw } \\
\text { Resist (mW) }\end{array}$ & \multicolumn{2}{|c|}{959.4} & \multicolumn{2}{|c|}{1072.5} & \multicolumn{2}{|c|}{3250} & \multicolumn{2}{|c|}{581.25} & \multicolumn{2}{|c|}{817.5} & \multicolumn{2}{|c|}{617.5} & 773 \\
\hline Diode (mW) & 4536 & 3888 & 7875 & 6750 & 6930 & 5940 & 962.5 & 875 & 2090 & 1900 & 2200 & 2000 & 645 \\
\hline $\begin{array}{l}\text { Total PW } \\
\text { Diode (mW) }\end{array}$ & \multicolumn{2}{|c|}{8424} & \multicolumn{2}{|c|}{14625} & \multicolumn{2}{|c|}{12870} & \multicolumn{2}{|c|}{1837.5} & \multicolumn{2}{|c|}{3990} & \multicolumn{2}{|c|}{4200} & 645 \\
\hline $\begin{array}{l}\text { Totals (D+R) } \\
(\mathrm{mW})\end{array}$ & 5052.6 & 4330.8 & 8452.5 & 7245 & 8680 & 7440 & 1206.25 & 1212.5 & 2407.5 & 2400 & 2407.5 & 2410 & 1418 \\
\hline $\begin{array}{l}\text { Grand Total } \\
(\mathrm{mW})\end{array}$ & \multicolumn{2}{|c|}{9383.4} & \multicolumn{2}{|c|}{15697.5} & \multicolumn{2}{|c|}{16120} & \multicolumn{2}{|c|}{2418.75} & \multicolumn{2}{|c|}{4807.5} & \multicolumn{2}{|c|}{4817.5} & 1418 \\
\hline Current (mA) & 210 & 180 & 350 & 300 & 350 & 300 & 50 & 50 & 100 & 100 & 100 & 100 & 55 \\
\hline $\begin{array}{l}\text { Grand Total } \\
\text { (mA) }\end{array}$ & \multicolumn{2}{|c|}{390} & \multicolumn{2}{|c|}{650} & \multicolumn{2}{|c|}{650} & 10 & & \multicolumn{2}{|c|}{200} & & & 55 \\
\hline Power Totals & \multicolumn{6}{|c|}{ UP 28.2 Watt } & \multicolumn{6}{|c|}{ Down 25 Watt } & $1.4 \mathrm{~W}$ \\
\hline $\begin{array}{l}\text { Power Grand } \\
\text { Total }\end{array}$ & \multicolumn{13}{|c|}{ 54.7 Watt } \\
\hline $\begin{array}{l}\text { Current } \\
\text { Totals }\end{array}$ & \multicolumn{6}{|c|}{$1.16 \mathrm{~A}$} & \multicolumn{6}{|c|}{$1.03 \mathrm{~A}$} & $0.55 \mathrm{~A}$ \\
\hline $\begin{array}{l}\text { Current } \\
\text { Grand Total }\end{array}$ & \multicolumn{13}{|c|}{$2.245 \mathrm{~A}$} \\
\hline
\end{tabular}


During the design, two types of dimming where considered: analog and PWM. With analog dimming, $\mathrm{X} \%$ brightness is achieved by applying $\mathrm{X} \%$ of the maximum current to the LED; note however, that the relationship between current and output intensity is not perfectly linear. The main downside of this process is a shift in the peak output wavelength of the LEDs with changing input current. Dimming via PWM is accomplished by applying full current to the LED at a reduced duty cycle. For $\mathrm{X} \%$ brightness, full current is applied at an X\% duty cycle. The frequency of the PWM signal must be above $100 \mathrm{~Hz}$ to ensure that the PWM pulsing is not visible to the human eye. The MPHIS grow light intensity was controlled by PWM using a NI cFP-PWM-520 module. Table 3 presents grow light power consumption information for each particular LED set.

Figure 11. Electrical schematic for B5b-435-TL. Each $33 \mathrm{ohm}$ resistor dissipates $82.5 \mathrm{~mW}$. Together, all resistors dissipate $1072.5 \mathrm{~mW}(577.5 \mathrm{~mW}$ up \& $495 \mathrm{~mW} \mathrm{dw})$. Together, the diodes dissipate 14625 $\mathrm{mW}(7875 \mathrm{~mW}$ up \& $6750 \mathrm{~mW} \mathrm{dw})$. Total power dissipated by the array is $15697.5 \mathrm{~mW}(8452.5 \mathrm{~mW}$ up \& $7245 \mathrm{~mW} \mathrm{dw}$ ). The array draws current of $650 \mathrm{~mA}$ from the source. ( $350 \mathrm{~mW}$ up \& $300 \mathrm{~mW}$ $\mathrm{dw}$ ). Where up and dw (down) represent the LED circuits for the upper and lower half of the PCB respectively.

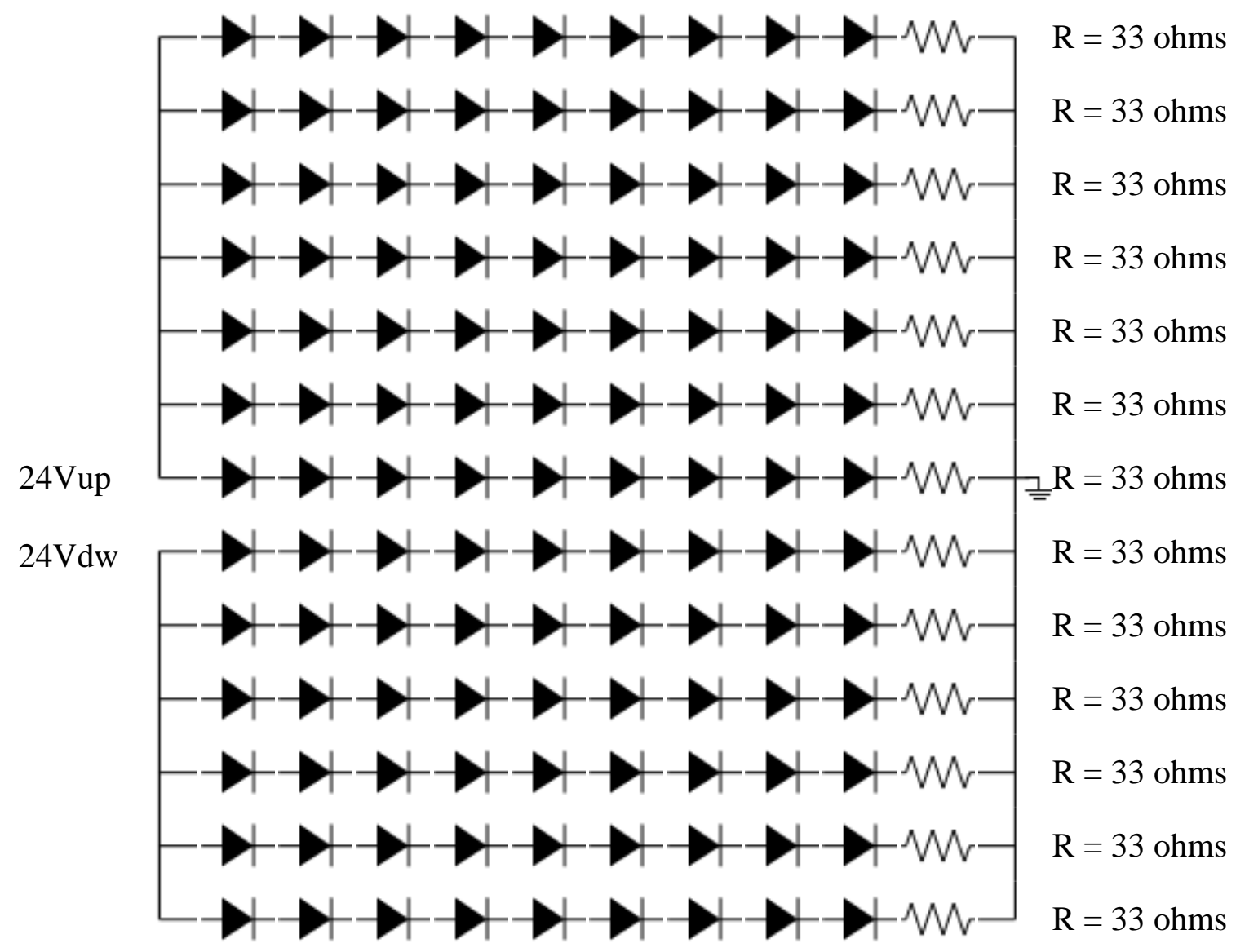




\subsection{Fluorescent Excitation Lights}

Excitation LEDs are powered using the NI-1744 smart camera current source output. LEDs are current-driven devices whose illumination is proportional to their current. Their current can be controlled in two ways. The first method, which was used to control the grow light board, is to use the V-I curve of each LED to determine the most appropriate ballast resistor to ensure the desired current through a series of LEDs (in this case input voltage was 24 VDC). This technique has a major drawback in that any variation in LED voltage causes a change in LED current and consequently a variation in the output intensity. Although less critical for the grow lights, the requirement for consistent excitation intensity is essential since the imager is designed to capture fluorescence, and variation in excitation energy will result in a corresponding variation in fluorescence intensity. The preferred method of regulating LED current is to drive LEDs with a constant-current source. The current source is constant and eliminates changes in current due to variations in voltage, which translates into constant LED brightness. However in the case of the excitation LEDs, this generates a potential problem as M-PHIS uses a combination of LEDs connected in series and parallel with the same current applied regardless of the number of LEDs (LED chain length). If one of the LED chains opens up (e.g. due to a LED failure) the LEDs in the remaining chains will see an increase in current flowing through them, changing their output and potentially resulting in the LEDs being driven out of specification and failing. The excitation lights shown in Figure 7 are two sets of four parallel rows of six LEDs in series. Both sets are Roithner LEDs (B56L5111P and LED450-01) with forward current ratings of $50 \mathrm{~mA}$. The camera constant current source is set to its maximum of $400 \mathrm{~mA}$ (8 rows x 50 $\mathrm{mA}$ ) and dissipates a total of $9.36 \mathrm{~W}$ during an excitation sequence. Figure $9 \mathrm{C}$ shows the powered excitation light and installed short pass $(490 \mathrm{~nm})$ filter that ensures no overlap of the incident excitation light occurs with the emitted fluorescence, which would subsequently negatively influence the desired measurement signal.

\subsection{M-PHIS Software}

Communication between the NI camera and NI controller was via Ethernet and IP protocols through shared variables. The main controller was coded with LabVIEW and the camera was programmed with Vision Builder for Automated Inspection (VBAI). The variables were initialized at the start of the main controller, and then commands, status and other information is sent back and forth via the network shared variables.

Figure 12 presents the M-PHIS software control panel: grow light intensity was controlled by setting the PWM value (0 to $100 \%$ ); the lighting schedule could be set to start and end twice any time during the day or stay on for $24 \mathrm{~h}$. The time and frequency of the image capture sequence could also be set. The white lights and LCTF frequency could be set either manually or via shared variables. Figure 13 shows the VBAI Inspection State Diagram. When the Capture Sequence variable is set to Full, as in Figure 12, the VBAI State Diagram will follow the default (Figure 13) and the camera will capture five types of images:

(1) GFP: Automatically via shared variable, the grow lights are turned off, excitation lights are turned $\mathrm{ON}$ and the LCTF is set to $510 \mathrm{~nm}$ with a FWHM at $10 \mathrm{~nm}$. This image predominantly captures only GFP fluorescence. 
(2) RED: Same as the GFP except the LCTF is set to $630 \mathrm{~nm}$ with a FWHM at $20 \mathrm{~nm}$. This image predominantly captures only red chlorophyll fluorescence.

(3) NIR: Same as the two above except the LCTF is set to $730 \mathrm{~nm}$ with a FWHM at $20 \mathrm{~nm}$. This image predominantly captures only near infrared chlorophyll fluorescence.

(4) Black: All lights are off. This image is subtracted from the GFP image to remove certain CCD imperfections (e.g. bad pixels).

(5) White: The white lights are on. This image produces a regular 'white light' image.

All images files are saved in two formats. The Tagged Image File Format (TIFF) [24] is a flexible format that normally saves 8 bits or 16 bits of pixel depth per color ( 8 bits equal 256 level of gray in our case) and uses a lossless compression. For the implemented camera, the file size was $1.25 \mathrm{MB}$ per image when the resolution was set to $1280 \mathrm{x} 1024$. The second format was JPEG (Joint Photographic Experts Group) [25], a "lossy" compression method that compresses data by discarding (losing) some of it. The process aims to minimize the amount of data that needs to be transferred telemetrically, an important consideration for remote deployments of this imager and the primary reason why the MPHIS is configured to also save in the JPEG format. The camera stored the data on the cFP-2120 controller's 4 GB compact flash card with filenames of the following format; ddmmyy-HHMMSStype-Image-temp-Fxx-Bxx.tif (or jpg) where Fxx and Bxx are the temperatures of the front and back of the Petri plate at the time of the image capture. Temperature data is also saved to a separate text file at a one minute frequency. When the imager is physically accessible or communication bandwidth permits, TIFF images are downloaded from the imager and serve as the main dataset for scientific studies and in-depth image analysis. 
Figure 12. LabVIEW Multispectral Plant Health Imaging System control panel.

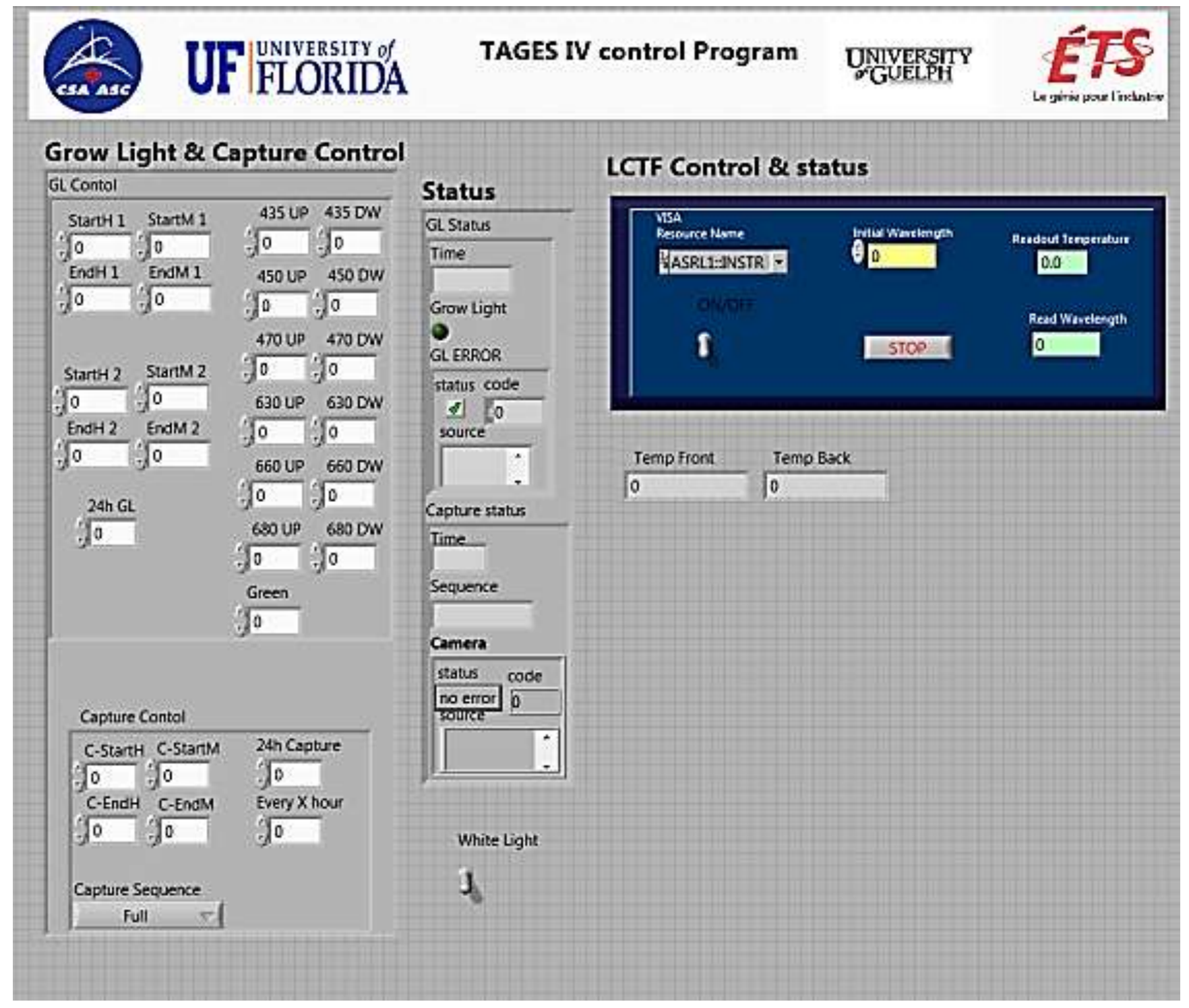

\subsection{Multispectral Plant Health Imaging System to Low-Pressure Chamber Interface}

The 24 VDC imager power was provided through an AC to DC adapter and the 120 VAC supply in the chamber interior. An Ethernet cable was also run into the chamber through a specially fabricated connector to permit real-time monitoring of the imager. The Ethernet cable connected the external laptop to the interior-chamber imager Ethernet hub. 
Figure 13. VBAI Inspection State Diagram (Multispectral Plant Imaging Health Imaging System capture sequence logic).

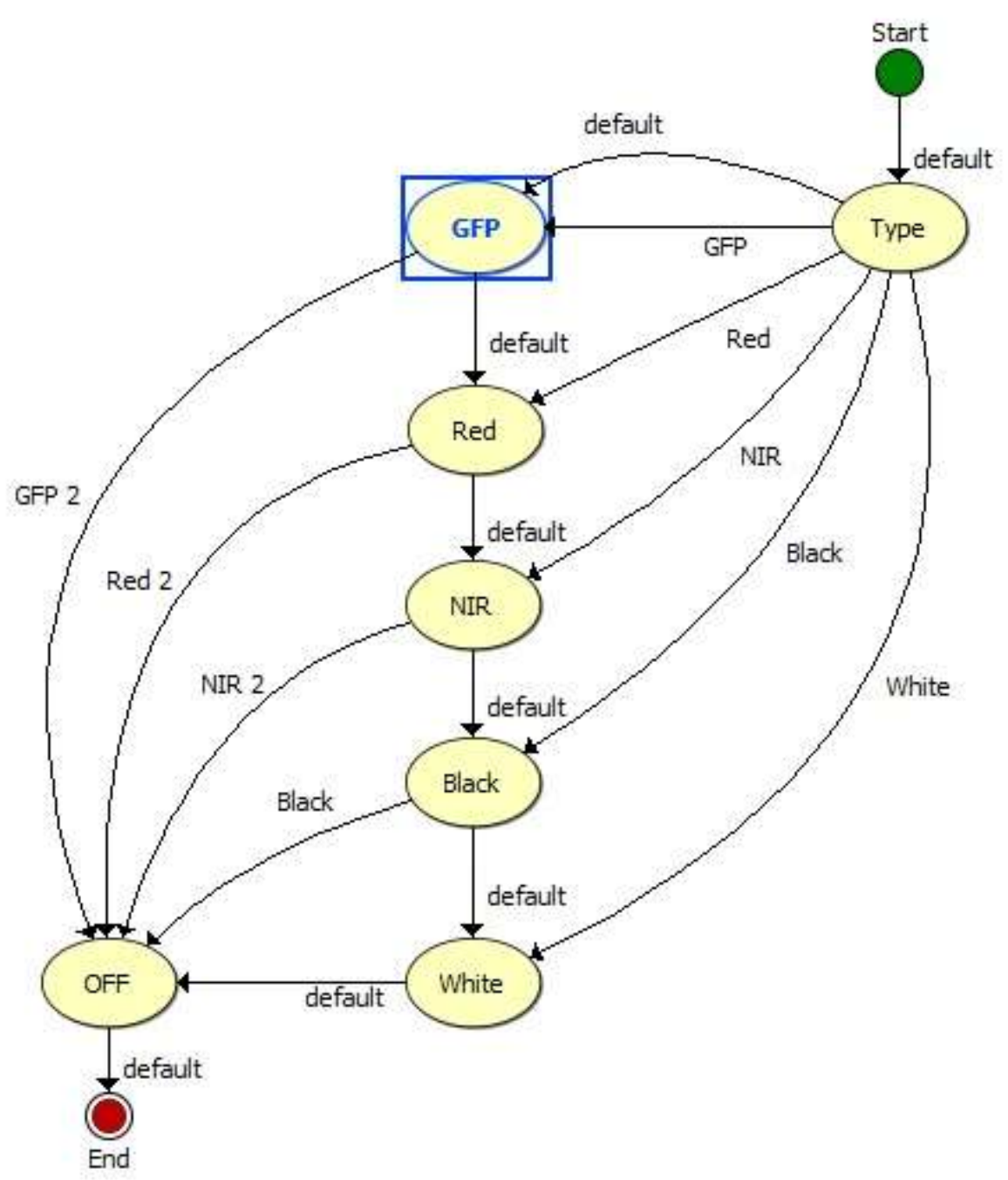

\section{Results and Discussion}

Like its predecessors [17, 18], M-PHIS underwent a series of basic laboratory tests before its deployment in a space analog environment. The output spectra of the excitation lights and grow lights were verified using a USB4000 Ocean Optics spectrometer. Current draw by the LEDs was measured using a Fluke 179 True RMS Multimeter. A Tektronix TDS3054B oscilloscope was used to authenticate the PWM grow lights voltage frequency, ratios and voltage output. After calibrating the lens to focus on the biological sample and adjusting the exposure time and gain of the camera to capture the maximum amount of fluorescence, tests were conducted to verify that only the emitted light from the sample was captured. In particular, several sequences were run without a Petri dish installed or with an empty Petri dish. The collected images were then examined and the GFP, Red and Infrared captures were dark, confirming that no excitation light entered into the imaging side of the system. 


\subsection{Short Duration Low Pressure Run}

The imager was installed in a hypobaric chamber at CESRF to evaluate the ability of the imager to capture images of induced GFP, natural red and near-infrared fluorescence and simulate plant health studies in a hypobaric environment. The testing was also designed to shed light on the operational constraints that a fully automated plant health imaging system may face on-orbit or other spaceflight scenarios.

Although the imager hardware and software went through several weeks of operational testing prior to deployment at CESRF, it was still unknown if the imager would operate at the low atmospheric pressures planned for the experiment. Such extreme operational conditions are far from standard, and as such no data was available regarding low pressure operation of each individual imager component. To address this question of operability under low pressure, a preliminary test was performed without biological samples in the imager. On February 16 2012, the imager was installed in the hypobaric chamber and set to capture a Full sequence (GFP, Red, InfraRed, Black and White) every 20 minutes under low pressure conditions. A calibration plate designed and fabricated by the University of Florida was installed in the imager for the tests Figure 14. The plate was engineered to fluoresce similar to GFP and chlorophyll. The chamber pressure was initially decreased from ambient to $25 \mathrm{kPa}$ over a 30 minute period and a full imaging sequence subsequently captured and verified in real-time. After confirmation of imager functionality at $25 \mathrm{kPa}$, the chamber pressure was further decreased to $5 \mathrm{kPa}$ over 10 minutes, where it was maintained for 30 minutes and a full sequence of images captured. The images obtained at low pressure $(25 \mathrm{kPa}$ and $5 \mathrm{kPa})$ were directly compared to those collected at ambient pressure and confirmed that the low pressures did not affect or alter the camera or LCTF optics in any significant way as shown in Figure 14. These tests also confirmed that the imager was capable of operating at low atmospheric pressures.

Figure 14. Capture sequences of the calibration plate at ambient pressure (top row) and at $5 \mathrm{kPa}$ (bottom row). These images confirm that reduced atmospheric pressure did not influence overall image quality, LCTF or other imager optics.

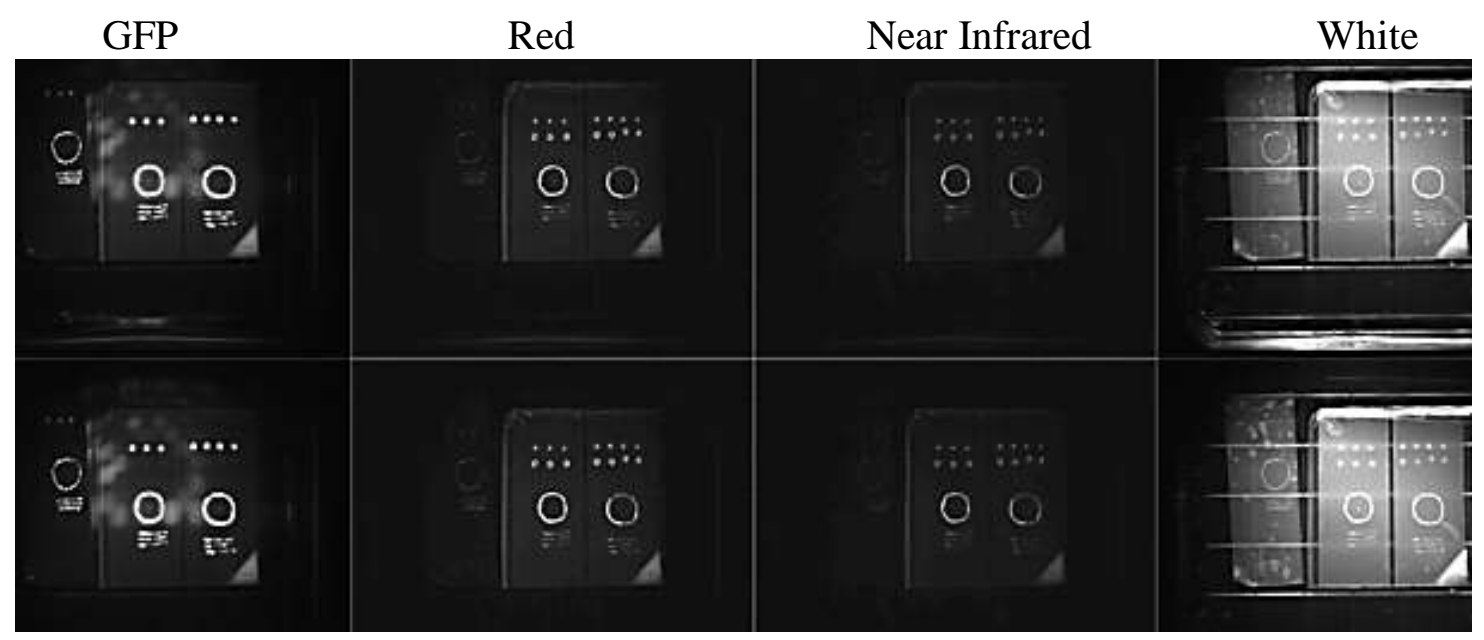




\subsection{Long Duration Low Pressure Run}

Following the general confirmation of M-PHIS functionality in low-pressure environments, a long duration low pressure run utilizing actual biological samples was conducted. After the chamber was closed for the first $5 \mathrm{kPa}$ run, M-PHIS was set to capture a sequence of images (GFP, Red, Infrared, Black, and White) at the start and every four hours thereafter for a period of forty-eight hours. This meant that the first capture sequence was taken when the chamber pressure was still at ambient. The chamber reached $5 \mathrm{kPa}$ 84-min following initiation of pressure draw-down. The second series of images, taken four hours from the start, was taken with the plants growing at $5 \mathrm{kPa}$. Figure 14 shows a comparison between the first two sequences. Two small rectangular markers are positioned on the top left of the sample tray and act as the LCTF calibration indicator. These reference indicators should nominally remain at the same intensity for each specific image capture type. For example, Figure 15 demonstrates that the intensity of the indicators were essentially the same for the image capture at atmospheric pressure and at $5 \mathrm{kPa}$.

During the long duration experiment, the internal temperature of M-PHIS rose beyond the tolerance limits of the biological samples contain within. After further investigation, several potential causes where discovered:

(1) Although the hypobaric chamber was temperature controlled, its lighting system composed of high-pressure sodium bulbs radiates heat on to the imager's metal casing (Figure 1). During the initial operability trial, the chamber lights were off (no biological samples in the chamber). During the plant imaging experiments however, the chamber lights were on to support control samples outside of M-PHIS.

(2) The imager cooling fans were insufficient in the hypobaric environment, as there was not enough air to carry away all the heat being generated. The preliminary operability tests were too short to allow the team to observe this heat management problem, which was further enhanced with the chamber lights were turned on.

(3) Since the imager system was sealed to prevent light contamination, waste heat and energy from the grow lights accumulated overtime.

To overcome or minimize the heat issue, the system was covered with a heat-reflecting blanket, the grow lights were kept off and the test durations were reduced. In the future it is likely that the fan located behind the sample tray will be replaced by a Peltier cooling system. The thermoelectric cooler could be controlled directly from the Compact FieldPoint PWM module. In addition, as the CESRF hypobaric chambers are equipped with crop irrigation lines, liquid-cooling loops could also be incorporated either individually or in combination with the thermoelectric cooler to further increase cooling capacity. 
Figure 15. Images sets from the first $5 \mathrm{kPa}$ hypobaric experiment. The sequence on the top is images captured at the beginning of the experiment where the pressure was still at ambient $(\sim 100 \mathrm{kPa})$. The series on the bottom were taken at $5 \mathrm{kPa}$ four hours later.

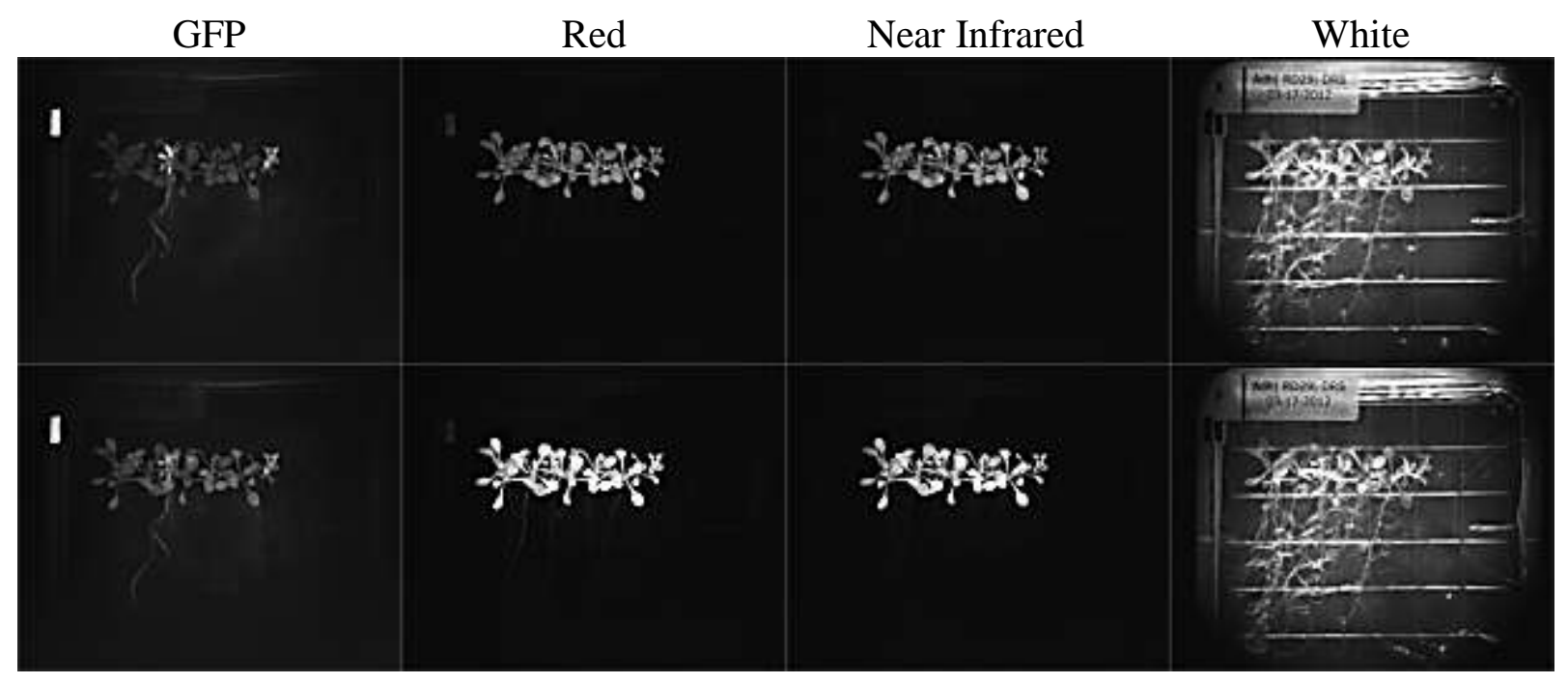

\section{Conclusions}

A plant health imaging system capable of capturing biological gene activities and translating these signals into plant stress measurements would be an indispensable diagnostic device in bioregenerative life support systems. In this study, we discuss the research and development of an autonomous multispectral imaging system designed to evaluate plant health in situ, in regular greenhouses or in plant production facilities located in hostile or space analog environments. With its LCTF the imager could be set to capture a series of images including, but not limited to GFP and natural chlorophyll fluorescence. In addition, its custom designed LED circuit board composed of independently controllable LEDs with seven distinct central wavelengths as well as independent control of the upper / lower half of the board meant the lighting system could be employed in a wide array of scenarios and studies. Numerous laboratory-based systems are available for capturing fluorescence but many are restricted to one or a predetermined set of filters. These systems also tend to involve considerable labour and do not include grow lights. Studies indicate that valuable information could be gathered with a system capable of measuring a wide range of fluorescent proteins as well as natural chlorophyll fluorescence $[6,9,10,11,12]$. Additionally, a system incorporating controllable photosynthetic lighting and thus able to maintain the plant samples for long duration experimental studies would provide even greater insights. Furthermore, plant research in space or in remote regions will likely be autonomous, include several biological sensors and will consist of robotic operations where telemetric data collection is a necessity because of restrictions in crew time and/or safety. The Multispectral Plant Health Imaging System was deployed in a hypobaric chamber and successfully captured images at several wavelengths, including those for GFP and red/near-IR for chlorophyll. In addition, M-PHIS was the first fluorescent imager to run autonomously in a low-pressure plant growth chamber. Its deployment and operations have demonstrated the feasibility of plant diagnostic systems that will allow for monitoring and control of space biology experiments and bioregenerative life support systems. Multispectral plant imaging systems are powerful tools for plant health monitoring and represent a significant step towards securing the technical capacity for sending plant heath information 
in a telemetric fashion from an extra-terrestrial location. Results from this work, combined with past and future experiments will be used to evaluate remote sensing plant response to low pressure environments.

\section{Acknowledgements}

The Canadian Space Agency founded the research and materiel to develop and build the imager. We thank Mr. Jamie Lawson from the University of Guelph's Controlled Environment Systems Research Facility. We also thank Pierre Lortie, Ralph Nolting and Maxime Pepin-Thivierge of the Canadian Space Agency machine shop for contributing to the mechanical design and for aiding in prototype construction. The authors recognize and thank Éric Gloutnay EMC \& Electronic Component Engineer at Canadian Space Agency for his support in LED PCB layout and fabrication. We also thank Nathalie Cassidy from the Canadian Space Agency for her important logistical contributions to this work.

\section{References}

1. Wheeler, R.M. Plants for human life support in space: from Myers to Mars. Gravitational and Space Biology 2010, 23, 25-36.

2. Bamsey, M.; Graham, T.; Stasiak, M.; Berinstain, A.; Scott, A.; Vuk, T.R.; Dixon, M. Canadian advanced life support capacities and future directions. Advances in Space Research 2009, 44, 151-161.

3. Bamsey, M.; Berinstain, A.; Graham, T.; Neron, P.; Giroux, R.; Braham, S.; Ferl, R.; Paul, A.L.; Dixon, M. Developing strategies for automated remote plant production systems: Environmental control and monitoring of the Arthur Clarke Mars Greenhouse in the Canadian High Arctic. Advances in Space Research 2009, 44, 1367-1381.

4. Tamponnet, C.; Savage, C. Closed Ecological Systems. Journal of Biological Education 1994, 28, 167-174.

5. Plautz, J.D.; Day, R.N.; Dailey, G.M.; Welsh, S.B.; Hall, J.C.; Halpain, S.; Kay, S.A. Green fluorescent protein and its derivatives as versatile markers for gene expression in living Drosophila melanogaster, plant and mammalian cells. Gene 1996, 173, 83-87.

6. Manak, M.S.; Paul, A.-L.; Sehnke, P.C.; Ferl, R.J. Remote sensing of gene expression in Planta: transgenic plants as monitors of exogenous stress perception in extraterrestrial environments. Life Support Biosph Sci 2002, 8:83-91.

7. Paul, A.-L.; Murdoch, T.; Ferl, E.; Levine, H.G.; Ferl, R. The TAGES Imaging System: Optimizing a green fluorescent protein imaging system for plants. SAE Technical Paper 2003, 2003-01-2477.

8. Paul, A.-L.; Ferl, R.J. The biology of low atmospheric pressure - implications for exploration mission design and advanced life support. Gravitational and Space Biology 2006, 19, 3-18.

9. Baker, N.R.; Rosenquist, E. Applications of chlorophyll fluorescence can improve crop production strategies: an examination of future possibilities. Journal of Experimental Botany 2004, 55, 1607-1621.

10. Ehlert, B.; Hincha, D. Chlorophyll fluorescence imaging accurately quantifies freezing damage and cold acclimation responses in Arabidopsis leaves. Plant Methods 2008, 4, 12.

11. Galston, A.W. Photosynthesis as a basis for life support on Earth and in space: photosynthesis and transpiration in enclosed spaces. Bioscience 1992, 42, 490-493.

12. Lichtenthaler, H.K.; Babani, F. Detection of photosynthetic activity and water stressby imaging the red chlorophyll fluorescence. Plant Physiology and Biochemistry 2000, 38, 889-895.

13. Stewart, C.N., Jr. The utility of green fluorescent protein in transgenic plants. Plant cell reports 2001, 20, 376-382. 
14. Heim, R.; Tsien, R.Y. Engineering green fluorescent protein for improved brightness, longer wavelengths and fluorescence resonance energy transfer. Curr Biol 1996, 6, 178-182.

15. Corp, L.A.; McMurtrey, J.E.; Chappelle, E.W.; Kim, M.S.; Daughtry, C.S.T. In Optimal fluorescence excitation wavelengths for detection of stress in vegetation, Geoscience and Remote Sensing Symposium, 1996. IGARSS '96. 'Remote Sensing for a Sustainable Future.', International, 27-31 May 1996, 1996; 1996; pp. 1812-1815 vol.1813.

16. Wehkamp, C.A.; Stasiak, M.; Lawson, J.; Yorio, N.; Stutte, G.; Richards, J.; Wheeler, R.; Dixon, M. Radish (Raphanus sativa L. cv. Cherry Bomb II) growth, net carbon exchange rate, and transpiration at decreased atmospheric pressure and / or oxygen. Gravitational and Space Biology 2012, 26, 3-16.

17. Abboud, T.; Bamsey, M.; Paul, A.-L.; Graham, T.; Braham, S.; Noumeir, R.; Berinstain, A.; Ferl, R. Deployment of a Fully-Automated Green Fluorescent Protein Imaging System in a High Arctic Autonomous Greenhouse. Sensors 2013, 13, 3530-3548.

18. Paul, A.-L.; Bamsey, M.; Berinstain, A.; Braham, S.; Neron, P.; Murdoch, T.; Thomas, G.; Ferl, J.R. Deployment of a prototype plant GFP imager at the Arthur Clarke Mars Greenhouse of the Haughton Mars Project. Sensors 2008, 8, 2762-2773.

19. Folta, K.; Koss, L.; McMorrow, R.; Kim, H.-H.; Kenitz, J.D.; Wheeler, R.; Sager, J. Design and fabrication of adjustable red-green-blue LED light arrays for plant research. BMC Plant Biology 2005, 5, 17.

20. Goins, G.D.; Yorio, N.C.; Sanwo, M.M.; Brown, C.S. Photomorphogenesis, photosynthesis, and seed yield of wheat plants grown under red light-emitting diodes (LEDs) with and without supplemental blue lighting. Journal of Experimental Botany 1997, 48, 1407-1413.

21. Porra, R.J.; Thompson, W.A.; Kriedemann, P.E. Determination of accurate extinction coefficients and simultaneous equations for assaying chlorophylls $\mathrm{a}$ and $\mathrm{b}$ extracted with four different solvents: verification of the concentration of chlorophyll standards by atomic absorption spectroscopy. Biochimica et Biophysica Acta (BBA) - Bioenergetics 1989, 975, 384394.

22. Schurr, U.; Walter, A.; Rascher, U. Functional dynamics of plant growth and photosynthesis-from steady-state to dynamics--from homogeneity to heterogeneity. Plant Cell Environ 2006, 29, 340-352.

23. West-Eberhard, M.J.; Smith, J.A.; Winter, K. Plant science. Photosynthesis, reorganized. Science 2011, 332, 311-312.

24. Wikipedia, t.f.e. Tagged Image File Format. Available online: http://en.wikipedia.org/wiki/Tagged_Image_File_Format\#cite_ref-0 (5 september 2012),

25. Wikipedia, t.f.e. JPEG. Available online: http://en.wikipedia.org/wiki/JPEG (5 september 2012).

(C) 2013 by the authors; licensee Insciences Journal. 